Y. Miyata

Nagoya Math. J.

Vol. 111 (1988), 165-171

\title{
ON THE ISOMORPHISM CLASS OF THE RING OF ALL INTEGERS OF A CYCLIC WILDLY RAMIFIED EXTENSION OF DEGREE $p$ II
}

\author{
YOSHIMASA MIYATA
}

Let $k$ be an algebraic number field with the ring of integers $\mathfrak{o}_{k}=\mathfrak{0}$ and let $G$ be a cyclic group of order $p$, an odd prime. Let $K / k$ be a cyclic extension of degree $p$ with the ring of integers $\mathfrak{D}_{K}$. Then, $\mathfrak{D}_{K}$ is an $\mathfrak{Q} G$ module. In the case that $K / k$ is tamely ramified, L. McCulloh [3] proved that the subset $R(\mathrm{o} G)$ of the classes $\mathrm{cl}(\mathcal{D})$ of the rings $\mathscr{D}$ in the class group $\mathrm{Cl}^{0}(a G)$ is equal to the subgroup $\mathrm{Cl}^{0}\left({ }^{\circ} G\right)^{J}$ generated by all $c^{a}, c \in$ $\mathrm{Cl}^{\circ}\left({ }^{\circ} G\right), a \in J$, where $J$ denotes the Stickelberger ideal (for the definitions, see below).

Now, in the previous paper [4], we studied the case that $K / k$ is wildly ramified. Let $\Gamma(\bigcap)$ be the genus containing $\bigcirc$. From H. Jacobinski's results [2], we know that there exists a one-to-one corresponding between the isomorphism classes in $\Gamma(\mathcal{O})$ and the elements of the class group $M$ (for the definition, see also below). The group $\Delta$ of automorphisms of $G$ acts on $M$ and so $M^{J}$ can be defined as in the group $\mathrm{Cl}^{\circ}\left({ }_{0} G\right)$. In [4], we defined the invariant $N(\mathcal{D})$ which is an element of $M$, and showed that $N(\mathfrak{D}) \in M^{J}$ (cf. [4, Theorem 4]). The purpose of this paper is to prove that the subset $R_{w}(\mathrm{o} G)$ of invariants $N(\mathcal{O})$ of the rings $\subseteq$ in the wildly ramified extensions $K / k$ of degree $p$ is equal to $M^{J}$ (Theorem 3).

Let $g$ be a fixed generator of $G$ and $\zeta$ be a primitive $p$-th root of unity. Throughout this paper, we assume that $k$ contains $\zeta$. In Section 1 , we shall recall the definitions given in [4], and prove Theorem 1 which is the modification of Theorem 4 of [4]. In Section 2, we shall recall L. McCulloh's results [3] and define a $\Delta$-homomorphism $\psi$ from $\mathrm{Cl}^{0}(\mathfrak{o} G)$ onto $M$. This homomorphism $\psi$ plays the important role in the proof of Theorem 3 that $R_{w}(\mathrm{D} G)=M^{J}$, which is proved in Section 3 .

Received February 16, 1987. 
$\S 1$.

Let $K / k$ be a cyclic wildly ramified extension of degree $p$ and let $G$ be a cyclic group of order $p$. We can view $G$ as Galois group $G(K / k)$ of $K / k$. In this section, we call definitions and Theorem 4 of [4]. For a prime ideal $\mathfrak{p}$ of $\mathfrak{o}$, let $k_{\mathfrak{p}}$ be the $\mathfrak{p}$-adic completion of $k$ with the valuation ring $\mathfrak{o}_{\mathfrak{p}}$, and let $K_{\mathfrak{p}}=k_{\mathfrak{p}} \otimes_{k} K$ and $\mathfrak{O}_{\mathfrak{p}}=\mathfrak{o}_{\mathfrak{p}} \otimes_{\mathfrak{o}} \mathfrak{O}$. Denote by $\pi(\mathfrak{p})(=\pi)$ and $e(p)(=e)$ a prime element and the absolute ramification index of $k_{\mathfrak{p}}$, respectively. We denote by $c(p)$ the ramification number of $K_{p} / k_{p}$. Then, it is well known that $-1 \leqq c(p) \leqq p e(\mathfrak{p}) /(p-1)$. Let $P_{1}=P_{1}(K)\left(P_{0}=P_{0}(K)\right)$ be a product $\Pi \mathfrak{p}$ of $\mathfrak{p}$ such that $\mathfrak{p} \mid(p)$ and $0<c(\mathfrak{p})<p e(\mathfrak{p}) /(p-1)-1$ $(c(\mathfrak{p})=-1)$, respectively, and let $P=P_{0} P_{1}$. As in [4], define integers $d(p)$ by

$$
d(\mathfrak{p})= \begin{cases}p e(\mathfrak{p}) /(p-1)-c(\mathfrak{p}) & \text { for } \mathfrak{p} \mid P_{1} \\ p e(\mathfrak{p}) /(p-1) & \text { for } \mathfrak{p} \mid P_{0},\end{cases}
$$

Moreover, for $0 \leqq i<p$, integers $m_{i}(\mathfrak{p})$ are defined by

$$
m_{i}(\mathfrak{p})=[i d(\mathfrak{p}) / p]
$$

where $[x]$ denotes an integer with $[x] \leqq x<[x]+1$.

Now, we define $\mathfrak{O}_{p} G$-modules $L_{\mathfrak{p}}$ and an $\mathfrak{D} G$-module $L$. Let $E_{i}$ be an primitive idempotent of $k G$ with $g E_{i}=\zeta^{i} E_{i}$ for $0 \leqq i<p$. For $0<i<p$ and $\mathfrak{p} \mid P_{1}$, let

$$
a_{i}(\mathfrak{p})=\pi(\mathfrak{p})^{-m_{i}}\left(\sum_{j=0}^{i}\left(\begin{array}{l}
i \\
j
\end{array}\right)(-1)^{i-j} E_{j}\right)
$$

and $a_{0}(p)=1$. We define $\mathfrak{o}_{p} G$-modules $L_{\mathrm{p}}$ as follows:

(a) For $\mathfrak{p} \nmid P, L_{\mathfrak{p}}=\mathfrak{o}_{\mathfrak{p}} \otimes\left(\sum \circ E_{\imath}\right)$.

(b) For $\mathfrak{p} \mid P_{1}, L_{\mathfrak{p}}=\sum \mathfrak{o}_{\mathfrak{p}} a_{i}(\mathfrak{p})$.

(c) For $\mathfrak{p} \mid P_{0}, L_{\mathfrak{p}}=(1 / p) \mathfrak{o}_{\mathfrak{p}} G$.

Then, it is easily known that there exists an ${ }^{\circ} G$-module $L$ in $k G$ such that $\mathfrak{o}_{\mathfrak{p}} \otimes_{\mathfrak{o}} L=L_{\mathfrak{p}}$ for each $\mathfrak{p}$ (for example, see [5, p. 70 (5.3) Theorem]). Denote by $\Gamma(L)$ a genus including $L$. By the definition of $L$, we have $\mathfrak{D} \in \Gamma$ (cf. [4, Lemma 7]).

Next, we define a class group $M$. Let $\chi$ be a character of $G$ with $\chi(g)=\zeta$ and $X=\left\{\chi, \chi^{2}, \cdots, \chi^{p-1}\right\}$. Let $I$ be the group of fractional ideals of $k$ relatively prime to $P$ and let $\operatorname{Map}(X, I)$ be the group of functions from $X$ into $I$. As in [3], an automorphism $\delta$ in $\Delta(=$ Aut $G$ ) acts on $X$ and $\operatorname{Map}(X, I)$ as follows: 


$$
\chi^{i \delta}(g)=\chi^{i}\left(g^{\delta-1}\right) \quad \text { and } n^{\delta}\left(\chi^{i}\right)=n\left(\chi^{i \delta-1}\right), n \in \operatorname{Map}(X, I) .
$$

Let $Z \Delta$ be the group ring over the ring of integers $Z$ and an element $\theta$ of $Z \Delta$ be

$$
\theta=\sum_{\delta \in \Delta} t(\delta) \delta^{-1}
$$

where $g^{\delta}=g^{t(\delta)}$ with $1 \leqq t(\delta)<p$. Let the Stickelberger ideal $J$ of $Z \Delta$ be

$$
J=\left(p^{-1} \theta \cdot Z J\right) \cap Z \Delta .
$$

An element $a$ of $k G$ is written in the form;

$$
a=a_{0} E_{0}+a_{1} E_{1}+\cdots+a_{p-1} E_{p-1} .
$$

From [4, Lemmas 4 and 5], we have

LEMma 1. Let Aut $L_{p}$ be the group of $\mathfrak{o}_{p} G$-automorphisms of $L_{p}$. Then, if $a \in$ Aut $L_{\mathfrak{p}}$ for each $\mathfrak{p} \mid P, a_{0}, \cdots, a_{p-1}$ are P-units.

Let $H$ be defined by

$$
H=\left\{a \in k G \mid a \in \text { Aut } L_{\mathfrak{p}} \text { for } \mathfrak{p} \mid P \text { and } a_{0}=1\right\},
$$

and so by [4, Corollary 2], $H$ is $\Delta$-invariant. Then, we can define a $\Delta$ homomorphism $f$ from $H$ into $\operatorname{Map}(X, I)$ such that

$$
f(a)\left(\chi^{i}\right)=a_{i} \mathfrak{0} .
$$

The class group $M$ is defined by

$$
M=\operatorname{Map}(X, I) / f(H) .
$$

Lemma 2. For $n \in \operatorname{Map}(X, I)$, let cl $n$ denote a natural image of $n$ in $M$. Then, there exists an element $m$ of $\operatorname{Map}(X, I)$ such that $\left(m\left(\chi^{i}\right),(p)\right)=1$ and $\operatorname{cl} m=\operatorname{cl} n$.

Proof. Let $\Lambda$ be a left order of $L$ :

$$
\Lambda=\{a \in k G \mid a L \subseteq L\} .
$$

Let an ideal $\mathfrak{f}$ of $\mathfrak{o}$ be the order ideal of the factor module $\left(\sum \mathfrak{\circ} E_{i}\right) / \Lambda$ (for the definition, see [5, p. 49]). By the definition of $L$, the set of prime divisors of $f$ is the set of prime divisors of $P$. Let $S$ be

$$
S=\left\{a \in k G \mid a E_{i} \equiv 1(\mathfrak{f}) \text { for } 0<i<p\right\} .
$$

Then, by H. Jacobinski's results [2, p. 8], we have $H \supseteq S$. Every coset of 
the ray $R(\mathfrak{f}) \bmod \mathfrak{f}$ in $I$ contains infinite many primes (for example, see [4, p. 215]). Thus, we can choose ideals $m\left(\chi^{i}\right)$ such that $m\left(\chi^{i}\right)$ and $n\left(\chi^{i}\right)$ be in the same coset of $R(\mathfrak{f})$ in $I$ and $\left(m\left(\chi^{i}\right),(p)\right)=1$. Since $H \supseteq S$, cl $n=$ cl $m$, which completes the proof of Lemma 2 .

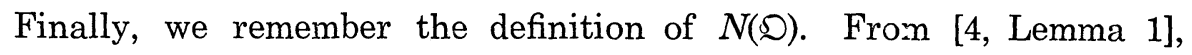
there exists an element $\alpha$ of $\mathfrak{D}$ such that $\alpha^{p} \in \mathfrak{D}$ and for $\mathfrak{p} \mid P$,

$$
\alpha^{p} \equiv 1\left(\pi(p)^{d(p)}\right) .
$$

Then, for $1 \leqq i<p$,

$$
\left(\alpha^{i p}\right)=\mathfrak{b}_{i} \mathfrak{c}_{i}^{-p},
$$

where $\mathfrak{b}_{i}$ is a $p$-power free integral ideal and $\mathfrak{c}_{i}$ is a fractional ideal. By

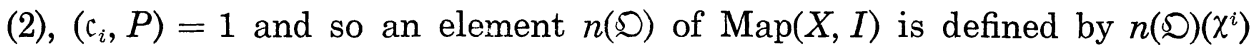

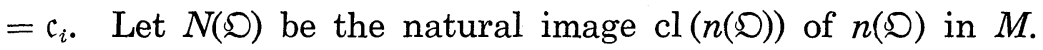

From [4, Theorem 4], we have the following theorem.

THEOREM 1. Let $K / k$ be a wildly ramified extension of degree $p$ with the discriminant $\operatorname{dis}(K / k)$. Let $L$ and $M$ be as above, and let $J$ be the Stickelberger ideal in $Z \Delta$. Then,

(i) $N(@) \in M^{J}$ and

(ii) for given ideal $\mathfrak{a}$ of $\mathfrak{b}$ with $(\mathfrak{a},(p))=1$, there exists a wildly ramified extension $K^{\prime} / k$ of degree $p$ such that $\mathfrak{O}^{\prime} \in I(L)=\Gamma(\bigcirc)$ and $\left(\operatorname{dis}\left(K^{\prime} \mid k\right), \mathfrak{a}\right)=1$.

Proof. (i) of Theorem 1 is Theorem 4 of [4] and hence its proof is done. Next, we prove (ii). Taking sufficiently large integers $n(p)$ for $\mathfrak{p} \mid \mathfrak{a}(p)$, we choose an element $b$ of $\mathfrak{o}$ such that for $\mathfrak{p} \mid(p), b \equiv \alpha^{p}\left(\pi(\mathfrak{p})^{n(p)}\right)$ and for $\mathfrak{p} \mid \mathfrak{a}$,

$$
b \equiv 1\left(\pi(p)^{n(p)}\right)
$$

Let $\beta=\sqrt[p]{b}$ and $K^{\prime}=k(\beta)$. Then, we see that for $\mathfrak{p} \mid(p)$, the ramification number of $K^{\prime} / k$ is equal to the ramification number of $K / k$. Thus, by [4, Corollary 1], $\mathfrak{S}^{\prime} \in \Gamma(L)$. As in (3), let $\left(\beta^{p}\right)=\mathfrak{b c} c^{-p}$. Then, if $\mathfrak{p} \mid \operatorname{dis}\left(K^{\prime} / k\right)$ and $(\mathfrak{p},(p))=1, \mathfrak{p}$ is a prime divisor of $\mathfrak{b}$ (for example, see [1, p. 91 Lemma 5]). By (4), $(\mathfrak{b}, \mathfrak{a})=1$ and so $\left(\operatorname{dis}\left(K^{\prime} / k\right), \mathfrak{a}\right)=1$, which completes the proof of Theorem 1.

$\S 2$.

In this section, we recall L. McCulloh's results [3], Let $X^{\prime}=\left\{\chi^{0}\right\} \cup X$, 
and $I^{\prime}$ be the group of fractional ideals of 0 relatively prime to $(p)$. Let $\mathfrak{o}_{P}$ be the semilocalisation of $\mathfrak{D}$ at $p$, and denote by $u\left(\mathfrak{o}_{P} G\right)$ the group of units of the ring $\mathfrak{o}_{\nu} G$. We define a homomorphism $f$ from $u\left(\mathfrak{o}_{p} G\right)$ into $\operatorname{Map}\left(X^{\prime}, I^{\prime}\right)$ by

$$
f(a)\left(\chi^{i}\right)=\chi^{i}(a) \mathfrak{0} .
$$

Then, the class group $\mathrm{Cl}(\mathfrak{o} G)$ of $\mathfrak{o} G$ is isomorphic to the factor group $\operatorname{Map}\left(X^{\prime}, I^{\prime}\right) / f\left(u\left(\mathfrak{o}_{p} G\right)\right)$. We extend an element $n$ of $\operatorname{Map}\left(X, I^{\prime}\right)$ to an element of $\operatorname{Map}\left(X^{\prime}, I^{\prime}\right)$ by setting $n\left(\chi^{0}\right)=\mathfrak{D}$, and hence we can view $\operatorname{Map}\left(X, I^{\prime}\right)$ as a subgroup of $\operatorname{Map}\left(X^{\prime}, I^{\prime}\right)$. Let $\phi$ be the natural homomorphism from $\operatorname{Map}\left(X, I^{\prime}\right)$ into $\operatorname{Map}\left(X^{\prime}, I^{\prime}\right) / f\left(u\left(0_{p} G\right)\right)$. Then,

$$
\operatorname{Ker} \phi=\left\{f(a) \mid a \in u\left(\mathfrak{o}_{p} G\right) \text { and } a E_{0} \text { is a unit of } \mathfrak{o}\right\} \text {. }
$$

By [3, (2.3.2) Proposition], we have $\phi\left(\operatorname{Map}\left(X, I^{\prime}\right)\right)=\mathrm{Cl}^{\circ}(\mathfrak{o} G)$. Let $T$ be a subgroup of $u\left(\mathfrak{o}_{p} G\right)$ consisting of elements $a$ in $u\left(\mathfrak{o}_{p} G\right)$ with $a E_{0}=1$. Then, clearly, $f(T)=\operatorname{Ker} \phi$.

Lemma 3. Let $T$ be as above and $H$ be as in Section 1 . Then, $T \subseteq H$.

Proof. An element of $T$ is clearly an automorphism of $L_{p}$ for each $\mathfrak{p} \mid P$, and so $T \subseteq H$ by the definition of $H$.

Now, noting $I^{\prime} \subseteq I$, we have a $\Delta$-homomorphism $\psi^{\prime}$ from $\operatorname{Map}\left(X, I^{\prime}\right)$ into $\operatorname{Map}(X, I)$. Then, it follows that $\psi^{\prime}$ induces a $\Delta$-homomorphism $\psi$ from $\mathrm{Cl}^{\circ}(\mathrm{o} G)$ into $M$ since $T$ and $H$ are $\Delta$-groups. Then, we have

Lemma 4. $\psi\left(\mathrm{Cl}^{\circ}(\mathfrak{o} G)\right)=M$.

Proof. By Lemma 2, for $\operatorname{cl} n \in M$, there exists an element $m$ of $\operatorname{Map}(X, I)$ such that $\left(m\left(\chi^{i}\right),(p)\right)=1$ and $\operatorname{cl} n=\operatorname{cl} m$ in $M$. Then, $m \in$ $\operatorname{Map}\left(X, I^{\prime}\right)$ and so $\operatorname{cl} n=\psi(\operatorname{cl} m) \in \psi\left(\mathrm{Cl}^{\circ}(\mathfrak{o} G)\right)$.

Since $\psi$ is a $\Delta$-homomorphism, we have

Corollary 1. $\psi\left(\mathrm{Cl}^{0}(\mathfrak{o} G)^{J}\right)=M^{J}$.

We conclude this section with stating L. McCulloh's Theorem [3, (1.3.1) Theorem].

THEOREM 2. Let $G$ be a cyclic group of order $p$, and $J$ be the Stickelberger ideal. Define a subset $R(\mathfrak{o} G)$ of $\mathrm{Cl}^{\circ}(\mathfrak{o} G)$ by

$$
R\left({ }^{\circ} G\right)=\left\{\mathrm{cl}\left(\mathfrak{D}_{K}\right) \mid K \text { runs over the set of tame extensions of degree } p\right\} .
$$


Then, $R\left({ }^{\circ} G\right)=\mathrm{Cl}^{\circ}\left({ }^{\circ} G\right)^{J}$. Moreover, given $m \in \mathrm{Cl}^{\circ}\left({ }^{\circ} G\right)^{J}$ and an ideal $a$ of $\mathfrak{o}$, there exists a tame extension $K / k$ such that $(\operatorname{dis}(K / k), \mathfrak{a})=1$ and $\mathrm{cl}(\cong)=m$.

$\S 3$.

In this section, we prove Theorem 3, which is the aim of this paper.

THEOREM 3. Let $G$ be a cyclic group of order $p$ and $K$ be a wildly

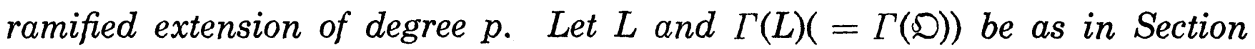
1. Define a subset $R_{w}\left({ }^{\circ} G\right)$ of $M$ by

$$
\begin{aligned}
R_{w}(\mathfrak{})= & \left\{N\left(\mathfrak{D}^{\prime}\right) \mid \mathfrak{D}^{\prime} \text { is the ring of a wildly ramified extension } K^{\prime} / k\right. \\
& \text { of degree } \left.p \text { with } \mathfrak{D}^{\prime} \in \Gamma(L)\right\} .
\end{aligned}
$$

Then, $R_{w}(\mathfrak{o} G)=M^{J}$. Moreover, given $m \in M^{J}$ and an ideal $\mathfrak{a}$ of $\mathfrak{o}$ with $(\mathfrak{a},(p))=1$, there exists a wildly ramified extension $K / k$ such that $(\operatorname{dis}(K / k), \mathfrak{a})=1$ and $N(\mathfrak{D})=m$.

Proof. By Theorem 1, we have $R_{w}(0 G) \subseteq M^{J}$. In the following, we have the existence of such a extension $K / k$ as above. By (ii) of Theorem 1 , there exists a wildly ramified extension $K^{\prime} / k$ such that (dis $\left.\left(K^{\prime} / k\right), \mathfrak{a}\right)$ $=1$ and $\mathscr{D}^{\prime} \in \Gamma(L)$. Let $\alpha^{\prime}$ be an element of $\varrho^{\prime}$ satisfying the congruences (2). Then, as in (3), we have

$$
\left(\alpha^{\prime i p}\right)=\mathfrak{b}_{i}^{\prime} c_{i}^{\prime-p} \quad \text { for } 1 \leqq i<p .
$$

As shown in the proof of Theorem $1,\left(\mathfrak{b}_{i}^{\prime}, \mathfrak{a}\right)=1$. Let $n=N\left(\mathfrak{D}^{\prime}\right)$ and $m^{\prime}$ $=n^{-1} m$ in $M$. Since $n \in M^{J}$ by Theorem 1 (i), we have $m^{\prime} \in M^{J}$. Then, by Corollary 1 , for some $\mathrm{cl}\left(\mathfrak{D}^{\prime \prime}\right) \in \mathrm{Cl}^{0}(\mathfrak{o} G)^{J} \psi\left(\mathrm{cl}\left(\mathfrak{D}^{\prime \prime}\right)\right)=m^{\prime}$. By Theorem 2, $S^{\prime \prime}$ can be chosen so that the discriminant of $k S^{\prime \prime}$ is relatively prime to the product $\mathfrak{b}$ of $\mathfrak{a}, \mathfrak{b}_{1}^{\prime}, \cdots, \mathfrak{b}_{p-1}{ }^{\prime}$. Moreover, as shown in the proof of $[3$, (4.2.1) Theorem], there exists an element $\beta$ of $\mathfrak{O}^{\prime \prime}$ such that $\beta^{p} \equiv 1\left((\zeta-1)^{p}\right)$. Let

$$
\left(\beta^{i p}\right)=\mathfrak{b}_{i} \mathfrak{c}_{i}^{-p},
$$

where $\mathfrak{b}_{i}$ is $p$-power free, and so $\left(\mathfrak{G}_{i}, \mathfrak{b}\right)=1$ because $\left(\operatorname{dis}\left(k \mathfrak{D}^{\prime \prime} \mid k\right), \mathfrak{b}\right)=1$. Ideals $\mathfrak{c}_{i}$ define an element $c$ of $\operatorname{Map}\left(X, I^{\prime}\right)$ by $c\left(\chi^{i}\right)=\mathfrak{c}_{i}$. By [3, (3.2.2) Theorem 3], $\operatorname{cl}\left(\mathfrak{D}^{\prime \prime}\right)=\mathrm{cl} c$, and hence

$$
m=\psi(\operatorname{clc} c) N\left(\Phi^{\prime}\right) .
$$

Now, let $F=k\left(\alpha^{\prime} \beta\right)$, and then $F$ is clearly the extension of degree $p$ over $k$. The action of $g$ on $\alpha^{\prime} \beta$ is defined by $g\left(\alpha^{\prime} \beta\right)=\zeta \alpha^{\prime} \beta$. Since $k(\beta) / k$ is 
tamely ramified, the ramification number $c^{\prime}(\mathfrak{p})$ of $F / k$ is equal to the ramification number $c(\mathfrak{p})$ of $K / k$ for $\mathfrak{p} \mid(p)$. Therefore, by [4, Corollary 1], the ring $\mathfrak{O}_{F}$ of all integers in $F$ belongs to the genus $\Gamma(L)$. We have

$$
\left(\alpha^{\prime} \beta\right)^{p i}=\mathfrak{b}_{i}^{\prime} \mathfrak{b}_{i}\left(\mathfrak{c}_{i}^{\prime} \mathfrak{c}_{i}\right)^{-p}
$$

and $\mathfrak{b}_{i}^{\prime} \mathfrak{b}_{i}$ is $p$-power free because $\mathfrak{b}_{i}^{\prime}$ and $\mathfrak{b}$ are $p$-power free with $\left(\mathfrak{b}_{i}^{\prime}, \mathfrak{b}_{i}\right)=1$. Then, ideals $\mathfrak{c}_{i}^{\prime}, \mathfrak{c}_{i}$ define an element $n\left(\mathfrak{O}_{F}\right)$ of $\operatorname{Map}(X, I)$ by $n\left(\mathfrak{D}_{F}\right)\left(\chi^{i}\right)=\mathfrak{c}_{i}^{\prime} c_{i}$,

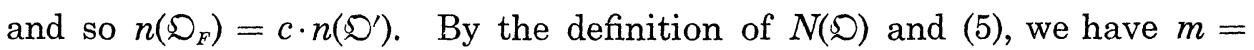
$N\left(\mathfrak{O}_{F}\right)$, which accomplishes the proof of Theorem 3.

\section{REFERENCES}

[1] J. W. S. Cassels and A. Fröhlich, "Algebraic Number Theory", Academic Press, London/New York, 1967.

[2] H. Jacobinski, Genera and decompositions of lattices, Acta Math., 121 (1968), $1-29$.

[ 3 ] L. R. McCulloh, A Stickelberger condition on Galois module structure for Kummer extensions of prime degree, in "Algebraic number fields", Proc. Durham Symp., Academic Press, London/New York, 1977, 561-588.

[4] Y. Miyata, On the isomorphism class of the ring of all integers of a cyclic wildly ramified extension of degree $p$, J. Algebra, to appear.

[5] I. Reiner, "Maximal orders", Academic Press, London, 1975.

Department of Mathematics

Faculty of Education

Shizuoka University

Shizuoka, 422 Japan 